\title{
A Method for Estimating Meanings for Groups of Shapes in Presentation Slides
}

\author{
Yuki Sakuragi, Atsushi Aoyama, Fuminori Kimura, and Akira Maeda
}

\begin{abstract}
This paper proposes a method for estimating the meanings for groups of shapes in presentation slides. The method of estimation is as follows; 1) Extracting features of each shape in a presentation slide, such as size, position, and type (e.g. circle, rectangle, arrow, etc.), 2) Analyzing the relations between these shapes, such as connection, inclusion, overlap, and similarity, 3) Grouping the shapes into meaningful groups using these relations, and estimating the meaning for each group, such as list, set, and flow/transition. The result of the proposed method could be used for retrieval and reuse of figures in presentation slides. In order to verify the effectiveness of the proposed method, we conducted an experiment of estimation accuracy, and achieved 0.93 in recall and 0.70 in precision.
\end{abstract}

\section{Index Terms-PowerPoint, OpenXML, figure retrieval.}

\section{INTRODUCTION}

A presentation tool such as Microsoft PowerPoint is a method of communicating information or opinions to other people. It is used in many situations, such as lectures and presentations in schools or universities, and meetings and conferences in companies. The number of publicly accessible presentation slides increases every year, and they could be regarded as a useful resource of knowledge.

There is a demand to raise working efficiency for making presentation slides, because drawing figures and diagrams usually requires a lot of time and effort, and presentation slides that are made in the past could be reused to save this time and effort. Therefore, search function for presentation slides, such as Microsoft PowerPoint, attracts much attention recently.

Figures, as well as text, are frequently used in presentation slides in order for the audience to easily and quickly understand the contents visually. Figures often summarize the contents of a slide, so they could be an important element to understand the contents of the slide.

Parts of figures in certain structures, such as flow/transition and diagrams, can be reused in other slides, and are actually frequently reused. It takes a lot of time and

Manuscript received September 5, 2014; revised November 17, 2014.

Y. Sakuragi is with at the Graduate School of Information Science and Engineering, Ritsumeikan University, 1-1-1 Noji-higashi, Kusatsu, Shiga 525-8577, Japan (e-mail: is0018ke@ ed.ritsumei.ac.jp).

A. Aoyama is with Graduate School of Technology Management, Ritsumeikan University, 1-1-1 Noji-higashi, Kusatsu, Shiga 525-8577, Japan (e-mail: aoyama@mot.ritsumei.ac.jp).

F. Kimura is with Kinugasa Research Organization, Ritsumeikan University, 56-1 Toji-in Kita-machi, Kita-ku, Kyoto, Kyoto 603-8577, Japan (e-mail: fkimura@ ed.ritsumei.ac.jp).

A. Maeda is with College of Information Science and Engineering, Ritsumeikan University, 1-1-1 Noji-higashi, Kusatsu, Shiga 525-8577, Japan (e-mail: amaeda@is.ritsumei.ac.jp). effort to make such figures from scratch. The working efficiency of making a presentation could be increased if we can reuse and modify figures that are made in the past. However, such figures are difficult to retrieve by using simple text search, because it depends on text in or around the figure to be retrieved.

We have proposed a presentation slide retrieval system which focuses on figures that consist of shapes [1]. In this system, a user formulates a query as a group of shapes, and the system retrieves slides that contain figures similar to the query. The disadvantages of the current system are that it requires a considerable amount of labor for formulating a query, and it does not consider the hierarchy of shapes

Estimating the meanings of groups of shapes from the hierarchy of shapes is especially important, because a hierarchical structure of shapes can express the meaning of shapes within a group of shapes, such as a list structure in a flow/transition.

Therefore, this paper proposes a method for estimating the meanings of groups of shapes from the hierarchy of shapes in presentation slides. The result of the proposed method could be used to retrieve figures in slides using the structure of shapes, and to reuse figures in presentation slides.

In this paper a "shape" represents a predefined basic shape, such as a circle, a rectangle, and an arrow. A "group of shapes" represents a certain set of shapes that are considered to have some meaning by the proposed system. Besides, a "figure" represents a figure or a diagram that consists of one or more groups of shapes and expresses some message to audience.

\section{RELATED WORK}

There is some research about presentation slides in the field of image processing [2], [3].

Wang et al. proposed an image classification system that focuses on synthetic (e.g. non-photographic) images [2]. The NPIC system extracts both content-based image retrieval features and metadata-based textual features for each image using machine learning. And the system classifies figures into block diagrams, tables, graphs and pie charts.

Liew et al. proposed a method for slide search using an image processing technique by regarding a slide as a picture [3]. They extracted three types of features from a picture, such as text features, image features and presentation features. The slide image type, that is one of the image features, is determined using the NPIC system.

There is also some research about image classification focused on specific synthetic image classes [4], [5].

Huang et al. proposed a system that aims at recognizing chart images using a model-based approach [4]. They 
classified a basic chart model into four different chart types based on their characteristics using basic object features and constraints between objects defined.

Carberry et al. proposed a method for extending document summarization to the summarization of information graphics [5]. They classified graphics and then extracted the data and the semantic meaning of several types of charts: such as bar, pie and line charts.

These researches are similar to our research, but our research does not regard a figure as a picture, and figures we focused on are not a table or a graph but basic shapes, such as a circle, a rectangle, and an arrow.

Zhou et al. proposed a method for extracting text from WWW images [6]. They detected text on images by examining connected components that satisfy certain criteria. It is similar to our research in the point of relating object and text, but our research uses only the information of shapes, so we can estimate the meaning of an object without using the metadata of the object.

Wang et al. proposed a generation method of presentation slides based on expression styles using slide structure [7], [8]. They derived the slide structure by focusing on the level of indentation of slide text. Their method derives the structure of multiple slides using text features, whereas our method derives the structure of a figure using the features of shapes.

Our proposed method estimates the meanings for groups of shapes by analyzing relations between shapes. In that sense, our method is different from existing works that analyze the structure of presentation slides.

Hayama et al. proposed a method for extracting the structure of information in presentation slides [9]. They organized each object on a slide into one of the attribute groups of "title", "figure", "table", "text", or "decoration", and proposed a method for structuring a slide by assembling the groups into a tree structure. However, their work deals with a different kind of structure from our research, because a "structure" in their work means a kind of attribute to the slides, but a "structure" in our work means the structure of a figure.

\section{Structure of Figures In PRESENTAtion Slides}

Shapes, such as circles, rectangles, lines, arrows, etc., are often used in presentation slides as constituent parts of figures/diagrams, because they make it easy to understand the contents of slides. Figures mostly consist of combination of many shapes, and these shape groups express the contents of a slide visually. Although a human can recognize a group of shapes as a figure having some meaning, it is difficult for a computer to do so. Therefore, the aim of this research is to let a computer estimate the meanings for groups of shapes as humans do.

\section{A. Grouping of the Shapes}

The proposed method groups the shapes in presentation slides that are considered to have some meanings. The "meanings" mentioned here are the meanings that are obtained by analyzing the questionnaire of recognition of the shape by humans.

We had a questionnaire to survey human's recognition for figures. We showed respondents some presentation slides that contain figures printed on papers. We asked the respondents to circle each group of shapes that is considered to have a meaning and to write the meaning of this group of shapes. Fig. 1 shows an example of an answer for the questionnaire.

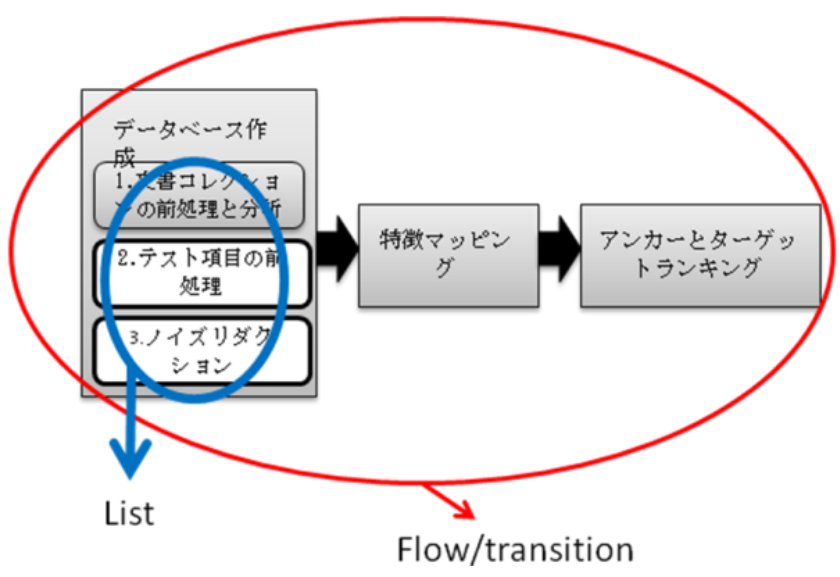

Fig. 1. An example of an answer for the questionnaire.

The number of the survey respondents is 8 and they are university students majoring in science and engineering. The number of presentataion slides we showed is 35 and these consist of slides from several different sources, i.e., from university lectures of science and liberal arts, and from business presentations by companies. We analyzed human's recognization for figures from the result of the questionnaire, and proposed a method for estimating meanings for groups of shapes in presentation slides. Besides, we obtained the correct answers for the experiments of estimation accuracy from the result of the questionnaire.

\section{B. The Hierarchical Structure of Shapes}

Figures often have a hierarchical structure. Fig. 2 shows an example figure that has a hierarchical structure. Such figures are often used in presentation slides.

If we look at this figure as a whole, the three gray quadrangles $\mathrm{A}, \mathrm{B}$, and $\mathrm{C}$ express a "flow" connected by arrows. In addition to that, this figure has a "list" structure within the quadrangle $\mathrm{A}$ at the left. Therefore, considering the hierarchical structure of a figure is important for estimating the meanings for groups of shapes.

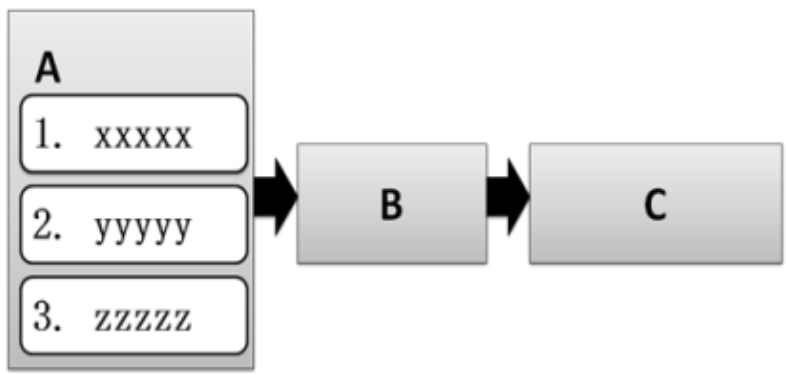

Fig. 2. An example of a figure that has a hierarchical structure.

\section{PROposed Method}

Fig. 3 shows the processing flow of the proposed method. First, we extract the features of each shape from the slide information expressed in XML. Second, we estimate the meaning of each group of shapes to analyze the relations between these shapes. Finally, we group the shapes into 
meaningful groups using these relations.

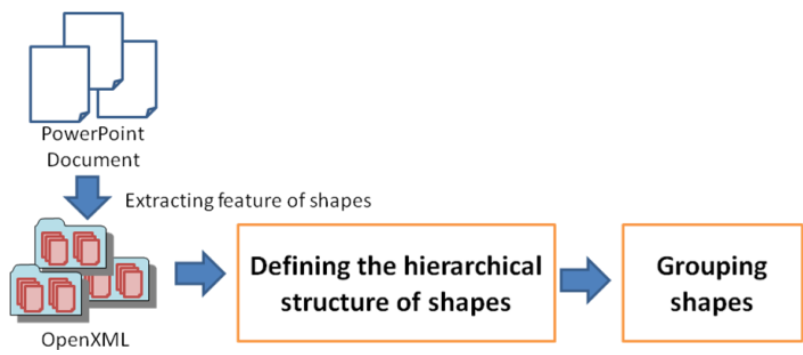

Fig. 3. Processing flow of the proposed method.

\section{A. Extraction of Shape Information}

The format for a presentation document with "pptx" extension is adapted by Microsoft Office 2007 and later versions. It can be converted into the OpenXML format that can be divided into texts and shapes.

It is easier for us to obtain the information of each element from an XML document, because it is text data which consists of tags, and arranged for every element. Fig. 4 shows a part of a pptx document that is converted into OpenXML. The information of the type of a shape, rotation angle, position, height and width, etc. can be extracted from this text data.

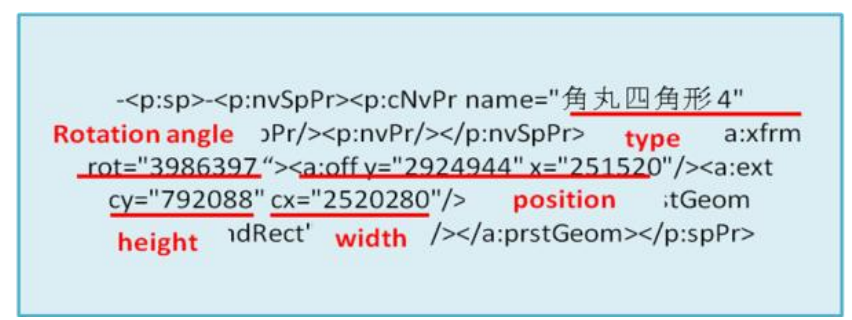

Fig. 4. An example of text information in an OpenXML document.

\section{B. Defining the Hierarchical Structure of Shapes}

In this research, we used inclusive relations between shapes in order to consider the hierarchy of shapes. The system assigns each shape to a proper level of hierarchy using inclusive relations.

In an example of Fig. 5, when the shape " $M$ " includes the shape " $N$ ", and if the hierarchy level of the shape " $M$ " is " $n$ ", the level of the shape " $N$ " is set to " $n+1$ ".

The shapes that are not included in any shape are set to "1st" hierarchy.

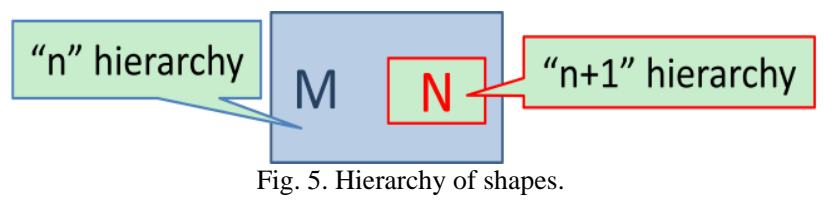

For example, as the result of defining the hierarchical structure of shapes to the presentation slide shown in Fig. 6, the system judges that the blue shape is the 1st hierarchy, the white shapes are the $2 \mathrm{nd}$, the yellow shapes are the $3 \mathrm{rd}$, and the pink shape is the 4 th.

The results of this process are used by the next step of grouping of shapes considering the meanings.

Also, using the results of the grouping step, we update the hierarchical structure. The grouped shapes constitute a new hierarchical class, and the shapes that belong to a group of shapes are assigned a hierarchical class one level below of the group of shapes.

Fig. 7 shows an example of the shapes and hierarchical structure of the shapes.

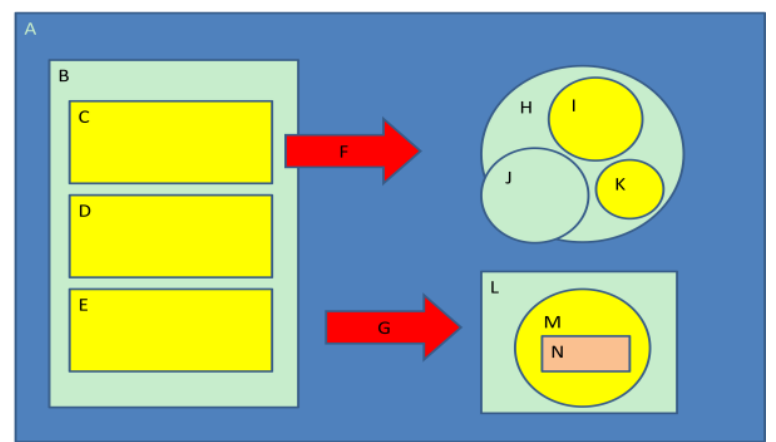

Fig. 6. An example of defining the hierarchical structure of shapes.

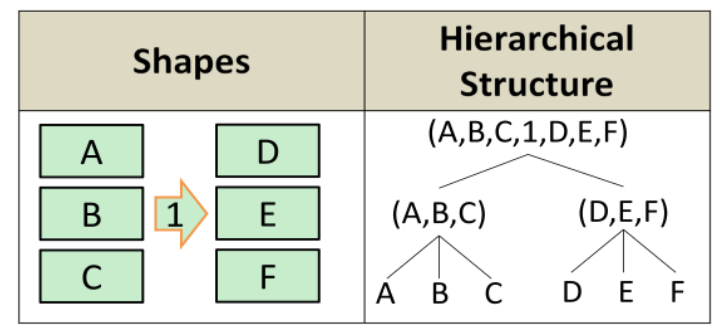

Fig. 7. An example of defining the hierarchical structure of shapes.

\section{Grouping of Shapes}

This step groups shapes into meaningful groups considering their meanings. In this paper, we adopt three meaningful features determined from the analysis of the questionnaire survey of human's recognition of figures in actual presentation slides, which was explained in Section III.A.

The three adopted meaningful features are as follows:

1) Shapes using arrows

2) Shapes that overlap

3) Shapes that have the same type and similar size

Using these meaningful features, we group the shapes into meaningful groups.

\section{1) Grouping as the meaning of a flow/transition}

The method determines which shape an arrow points to, and which shape an arrow comes from, and groups the arrow shape and the two shapes that are determined to be connected by that arrow. As shown in Fig. 8, this group of shapes has the meaning of "flow/transition" in which an arrow shape exists between shapes. This meaning is attached to the group of shapes that are grouped by an arrow. The meaning of "flow/transition" is used when the transition of procedure or things is expressed.

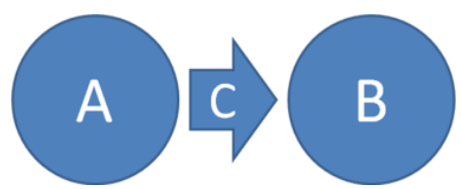

Fig. 8. An example of a group that has the meaning of "flow/transition".

In the grouping of meaning "flow", the system searches for the two shapes related by an arrow shape. The search range focuses on the shape which is within the angle of \pm 90 degrees in the direction of back to front of the arrow shape, as shown 
in Fig. 9. The system searches two shapes that are near to back and front of the arrow shape and groups these shapes.

The distance between the arrow shape and the target shape is defined as the shortest distance between the front point of the arrow shape and the boundary of the target shape, as shown in Fig. 9.

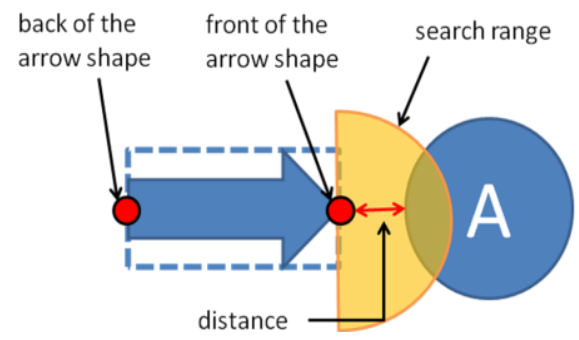

Fig. 9. The search range and distance of an arrow shape.

The system calculates all the distances between the arrow shape and all the shapes in the search range of the arrow and groups the arrow shape and the shape having the shortest distance. In the same way, the system finds a shape in the back of the arrow, and groups the shape and the arrow. The result of this method allows us to understand the meaning of a flow/transition between shapes correlated by an arrow.

\section{2) Grouping as the meaning of an overlap}

The method determines whether shapes overlap between each other, and groups the shapes that are determined to be overlapped. As shown in Fig. 10, this group of shapes has the meaning of "overlap" in which three circle shapes A, B, and $\mathrm{C}$ overlap with a doughnut shape behind them. This meaning is attached to the group of shapes that are grouped by overlapping. The meaning of "overlap" is used when an overlap, a correlation, and a hierarchical relationship are expressed.

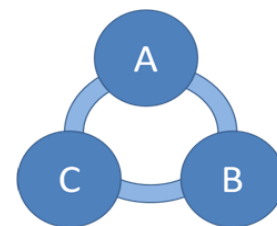

Fig. 10. An example of a group that has the meaning of "overlap".

In the grouping of the meaning "overlap", a figure is generalized to an ellipse or a rectangle. The system performs the following three kinds of overlap judgment: 1) rectangle and rectangle 2) rectangle and ellipse 3 ) ellipse and ellipse, as shown in Fig. 11.
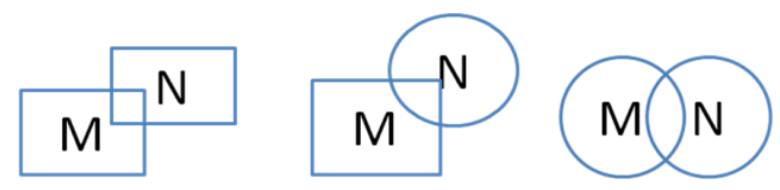

rectangle and rectangle rectangle and ellipse

ellipse and ellipse Fig. 11. An example of three kinds of overlap.

In the case of rectangle and rectangle, if at least one of four side lines of the rectangle $\mathrm{M}$ crosses at least one of four side line of the rectangle $\mathrm{N}$, the system judges the rectangle $\mathrm{M}$ and the rectangle $\mathrm{N}$ overlap.

In the case of rectangle and ellipse, if at least one of four side lines of the rectangle $M$ intersects the ellipse $N$, the system judges the rectangle $\mathrm{M}$ and the ellipse $\mathrm{N}$ overlap.

In the case of ellipse and ellipse, the system judges that they overlap when ellipses collide using the collision detection algorithm [10]. In the paper, Hwang et al. proposed an algorithm for collision detection with an ellipse and an ellipse. Their method transforms the collision detection with an ellipse and an ellipse into the collision detection with a point and an ellipse.

The flow of their method is as follows; 1) determine the target ellipse, 2) performs the scale transformation for two ellipses so that the target ellipse become exact circles with the radius of 1 unit length, 3) subtract 1 unit length of radius of the target circle and add 1 to the minor axis and the major axis of the other ellipse in order to make the collision detection with a point and an ellipse.

\section{3) Grouping as the meaning of a list}

The method determines whether shapes have the same type and are similar in size between each other, and groups the shapes that are determined so.

As shown in Fig. 12, this group of shapes has the meaning of "list" in which three rounded rectangle shapes have the same type and are similar in size. This meaning is attached to the group of shapes that are grouped by the same type and similar in size. The meaning of "list" is used when an itemized statement of information and parallel relations are expressed.

\section{1. $\mathrm{XXXXX}$ \\ 2. ууууу \\ 3. $\mathrm{zZZZZ}$}

Fig. 12. An example of a group that has the meaning of "list".
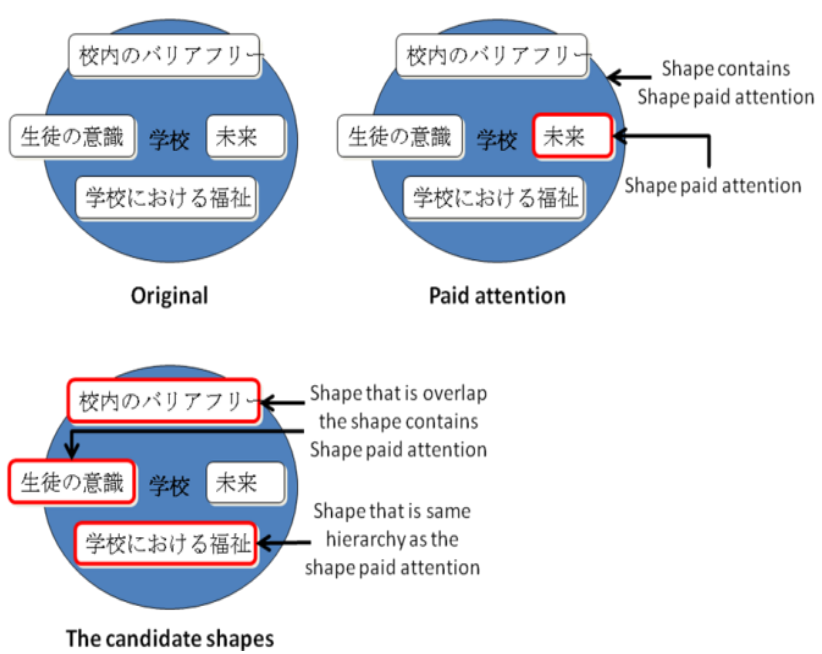

Fig. 13. An example of grouping as the meaning of a list.

In the grouping of the meaning "list", the system searches the same type and similar-sized shapes using the result of grouping in the meaning of an "overlap" and the result of defining the hierarchical structure of shapes.

The method of grouping in the meaning "list" is as follows;

1) The system selects a shape as the target shape.

2) The system determines the shapes to search for, which are the shapes within the same hierarchy, or if the target shape includes or overlaps with other shapes, the shapes that are included in or overlap with the target shape, as 
the candidate shapes for the judgment.

3) The candidate shape is regarded that it is contained in the "list" of the target shape, if it has the same type and similar in size as the target one. In this paper, if longitudinal width of a shape $\mathrm{M}$ is more than 0.5 times of longitudinal width of a shape $\mathrm{N}$ and less than 1.5 times of longitudinal width of a shape $\mathrm{N}$, the system groups these shapes into one.

Fig. 13 shows an example of grouping as the meaning of a list.

\section{EXPERIMENT}

TABLE I: THE ACCURACY RATE OF DEFINING THE HIERARCHICAL STRUCTURE OF SHAPES

Accuracy rate $\quad 0.76$

TABLE II: PRECISION AND RECALl OF EsTIMATING THE MEANINGS OF GROUPS OF SHAPES

\begin{tabular}{lll}
\hline \hline Meaning & Precision & Recall \\
\hline Flow/transition & 0.82 & 0.85 \\
Overlap & 0.59 & 1.00 \\
List & 0.68 & 0.95 \\
\hline Average & 0.70 & 0.93 \\
\hline \hline
\end{tabular}

In order to verify the effectiveness of the proposed method, we conducted an experiment of estimation accuracy. We computed the accuracy rate of defining the hierarchical structure of shapes, and precision and recall of estimating the meanings of groups of shapes.

The accuracy rate of defining the hierarchical structure of shapes and precision and recall of estimating the meanings of groups of shapes are defined as follows:

$$
\text { accuracy_rate }=\frac{|B|}{|C|}
$$

where:

- $|B|$ is the total number of the shapes whose hierarchy matched

- $|C|$ is the total number of the target shapes

$$
\begin{gathered}
\text { precision }=\frac{|R \cap A|}{|A|} \\
\text { recall }=\frac{|R \cap A|}{|R|}
\end{gathered}
$$

where:

- $|A|$ is the total number of the shape groups obtained by the proposed method

- $|R|$ is the total number of the correct shape groups

The correct answers were obtained from the result of a questionnaire survey conducted on 8 people.

The number of the slides that are used for the experiment is 18 , and the total number of shapes in these presentation slides is 187 . The slides are the same ones that were used in the questionnaire survey described in Section III.A.

\section{Results}

Table I shows the accuracy rate of defining the hierarchical structure of shapes. Table II shows the precision and recall of estimating the meanings of groups of shapes. We achieved 0.93 in recall and 0.70 in precision. However, the number of slides used in this experiment is not actually sufficient for reliable results, so we are planning to conduct a larger-scale experiment with more slides with more variations of shapes.

\section{DISCUSSION}

\section{A. Defining the Hierarchical Structure of Shapes}

Such as the slide on the right side of Fig. 14, white rectangles are arranged on four blue circles. Some of the rectangles are fully included in the blue circle, and others are partially stuck out from the circle. From the result of the questionnaire, we found that a human considers that all these rectangles are in the same group of elements and are semantically included in the blue circle. However, our proposed method cannot identify that these rectangles are in the same hierarchy, because it identifies that a shape is included in other shape only if one is geometrically fully included in others in the inclusion identification process.

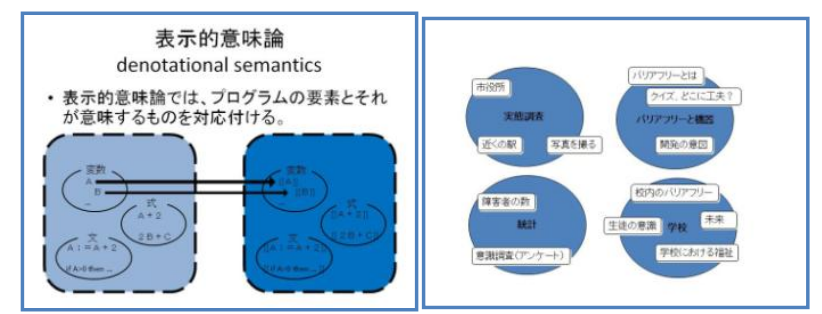

Fig. 14. Examples of slides that are actually used in the questionnaire survey.

In order to recognize that these rectangles are in the same hierarchy, it is necessary to consider an algorithm that allows some protrusion. In order for a better algorithm for building a hierarchy of shapes, it is necessary to consider how much protrusion should be allowed.

One of the possible solutions might be to use the area information of shapes. If the area of the shape " $\mathrm{A}$ " overlaps with the shape " $\mathrm{B}$ " more than 80 percent and the area of shape " $B$ " is far larger compared with the area of shape " $A$ ", the hierarchy of the shape " $A$ " could be regarded as the child hierarchy of the shape " $\mathrm{B}$ ".

\section{B. Estimating the Groups of Shapes}

A comparatively good result was obtained in estimating the meaning of grouped shapes in the experiment. However, using only three kinds of meanings are not enough for practical use. We would like to extend the algorithm so that it can estimate more variations of meanings. For example, from a flow chart, meanings such as circulation, branching, concentration, and emission should also be estimated.

An arrow shape connects not only individual shapes but also groups of shapes. However, the proposed method currently cannot estimate connections between groups of shapes. A possible solution to this problem is to group the shapes in two ways for estimating the meaning of "flow/transition", one with using the results of grouping for the meanings of "overlap" and "list", and another is not using them. If these results conflict, we can determine the feasible one by some evaluation function. In addition, an arrow shape sometimes connects a group that are grouped as the meaning 
of "flow/transition" and a shape or a group of some other meanings. Thus, the algorithm for grouping for the meaning of "flow/transition" is needed to be reconsidered.

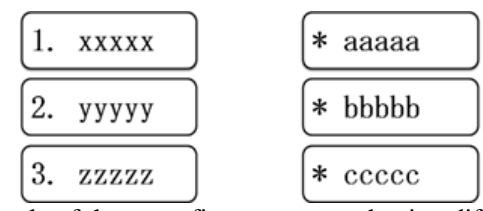

Fig. 15. An example of the same figure structure having different meanings.

\section{CONCLUSION}

This paper proposed a method for estimating the meanings for groups of shapes for the purpose of retrieving figures in presentation slides. The proposed method first estimates the hierarchy of shapes, and then groups the shapes in each hierarchy and estimates the meaning for each group. However, there are some issues to be considered, such as the order of grouping.

From the result of the questionnaire, we found that when humans estimate the meaning of a group of figure, they consider not only the shapes but also the text within or near the shapes. The group of shapes on the right side of Fig. 15 has the meaning of "list", but the group of shapes on the left side might have the meaning of "flow/transition" because the text of enumeration such as " 1 ", " 2 ", and " 3 " are contained in the rectangles. This indicates that text information is a very important element for estimating the meaning of a group of shapes. One of our future work is to consider text information, to make the estimation of the meaning for groups of shapes closer to human's estimation.

\section{REFERENCES}

[1] S. Tanaka, T. Tezuka, A. Aoyama, F. Kimura, and A. Maeda, "Slide retrieval technique using features of figures," in Proc. the International Multi-Conference of Engineers and Computer Scientists, Hong Kong, China, Mar. 2013, pp. 424-429.

[2] F. Wang and M. Y. Kan, "NPIC: hierarchical synthetic image classification using image search and generic features," in Proc. the 5th International Conference on Image and Video Retrieval, Tempe, Arizona, USA, 2006, pp. 473-482.

[3] G. M. Liew and M. Y. Kan,"Slide image retrieval: a preliminary study," in Proc. the 8th ACM/IEEE-CS Joint Conference on Digital Libraries, Pittsburgh, Pennsylvania, June 16-20, 2008, pp. 359-362.

[4] W. Huang, C. L. Tan, and W. K. Loew, "Model-based chart image recognition," in Proc. the International Workshop on Graphics Recognition, 2003, pp. 87-99.

[5] S. Carberry, S. Elzer, N. Green, K. McCoy, and D. Chester, "Extending document summarization to information graphics," in Proc. the ACL-04 Workshop, 2004, pp. 3-9.

[6] J. Zhou and D. Lopresti, "Extracting text from www images," in Proc. the 4th International Conference on Document Analysis and Recognition, 1997, vol. 1, pp. 248-252.
[7] Y. Wang and K. Sumiya, "A browsing method for presentation slides based on semantic relations and document structure for e-learning," Journal of Information Processing, vol. 20, no. 1, pp. 11-25, Jan. 2011

[8] Y. Wang and K. Sumiya, "A generation method of presentation slides based on expression styles using slide structure," in Proc. the 4th International Workshop with Mentors on Databases, Web and Information Management for Young Researchers, Nagoya, Japan, August 1, 2012.

[9] T. Hayama, H. Nanba, and S. Kunifuji, "Structure extraction from presentation slide information," in Proc. 10th Pacific Rim International Conference on Artificial Intelligence, 1987, pp. 2.2-1-2.2-6.

[10] K. S Hwang and M. D. Tsai, "On-line collision-avoidance trajectory planning of two planer robots based on geometric modeling," Journal of Information Science Engineering, vol. 15, 1999.

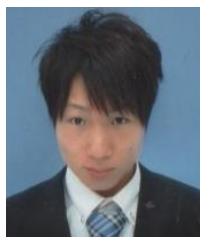

Y. Sakuragi is a master's student at the Graduate School of Information Science and Engineering, Ritsumeikan University. His research interests include multimedia information retrieval.

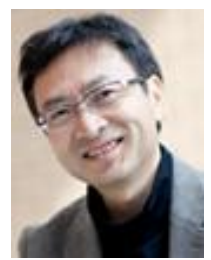

A. Aoyama is a professor at Graduate School of Technology Management, Ritsumeikan University. He obtained the Ph.D. degree in chemical engineering from Purdue University in 1994. His research interests include knowledge management, operational risk management, business intelligence (data and text mining) and big data, business model, business simulation, technology evaluation.

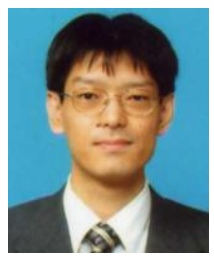

F. Kimura is a senior researcher at Art Research Center, Ritsumeikan University. He obtained the Ph.D. degree in engineering from Nara Institute of Science and Technology in 2007. His research interests include information retrieval, and text mining and multilingual information processing.

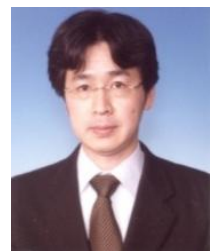

Akira Maeda is a professor at the Department of Media Technology, College of Information Science and Engineering, Ritsumeikan University. He received the B.A. and M.A. degrees in library and information science from University of Library and Information Science (ULIS) in 1995 and 1997, respectively, and received the Ph.D. degree in engineering from Nara Institute of Science and Technology (NAIST) in 2000 $\mathrm{He}$ has visited Virginia Polytechnic Institute and State University (Virginia Tech) from October 2000 through March 2001 as a postdoctoral visiting scholar. He has worked as a postdoctoral researcher of the CREST program, Japan Science and Technology Corporation (JST) from April 2001 through March 2002. He was a visiting professor at King's College London from September 2011 through September 2012. His research interests include digital libraries, digital humanities, information retrieval, and multilingual information processing. 